\title{
Personalized radiation dosimetry for PRRT_how many scans are really required?
}

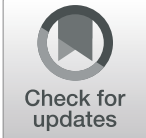

Nanette Freedman ${ }^{1 *}$, Mattias Sandström ${ }^{2 \dagger}$, Jonathan Kuten ${ }^{1}$, Natan Shtraus ${ }^{3}$, Inna Ospovat ${ }^{3}$, Albert Schlocker ${ }^{3}$ and Einat Even-Sapir ${ }^{1,4}$

* Correspondence: hanafre@tlvmc.
gov.il
${ }^{+}$Nanette Freedman and Mattias
Sandström are co-first authors.
${ }^{1}$ Institute of Nuclear Medicine, Tel
Aviv Sourasky Medical Center, 6
Weizman Street, 64239 Tel Aviv,
Israel
Full list of author information is
available at the end of the article

* Correspondence: hanafre@tlvmc. gov.i Sandström are co-first author ${ }^{1}$ Institute of Nuclear Medicine, Tel Aviv Sourasky Medical Center, 6 Weizman Street, 64239 Tel Aviv

Full list of author information is

\begin{abstract}
Purpose: Over recent years, peptide receptor radiotherapy (PRRT) has been recognized as an effective treatment for patients with metastatic neuroendocrine tumors (NETs). Personalized dosimetry can contribute to improve the outcome of peptide receptor radiotherapy (PRRT) in patients with metastatic NETs. Dosimetry can aid treatment planning, ensuring that absorbed dose to vulnerable normal organs (kidneys and bone marrow) does not exceed safe limits over serial treatments, and that absorbed dose to tumor is sufficient. Absorbed dose is estimated from a series of post-treatment SPECT/CT images. Total self-dose is proportional to the integral under the time activity concentration curve (TACC). Method dependence of imagebased absorbed dose calculations has been previously investigated, and we set out here to extend previous work by examining implications of number of data points in the TACC and the numerical integration methods used in estimating absorbed dose.

Methods: In this retrospective study, absorbed dose estimates and effective half-lives were calculated by fitting curves to TACCs for normal organs and tumors in 30 consecutive patients who underwent a series of 4 post-treatment SPECT/CT scans at $4 \mathrm{~h}$, $24 \mathrm{~h}, 4-5$ days, and 1 week following ${ }^{177}$ Lu-DOTATATE PRRT. We examined the effects of including only 2 or 3 rather than all 4 data points in the TACC, and the effect of numerical integration method (mono-exponential alone or in combination with trapezoidal rule) on the absorbed dose and half-life estimates. Our current method is the combination of trapezoidal rule over the first $24 \mathrm{~h}$, with mono-exponential fit thereafter extrapolated to infinity. The other methods were compared to this current method.

Results: Differences in absorbed dose and effective half-life between the current method and estimates based only on the second, third, and fourth scans were very small (mean differences $<2.5 \%$ ), whereas differences between the current method and 4-point mono-exponential fit were higher (mean differences $<5 \%$ ) with a larger range. It appears that in a 4-point mono-exponential fit the early $(4 \mathrm{~h})$ time point may skew results, causing some large errors. Differences between the current method and values based on only 2 time points were relatively small (mean differences $<3.5 \%$ ) when the $24 \mathrm{~h}$ and 1 week scans were used, but when the $24 \mathrm{~h}$ and $4-5$ days scans, or the $4-5$ days and 1 week scans were used, differences were greater.

(Continued on next page)
\end{abstract}

\section{SpringerOpen}

(c) The Author(s). 2020 Open Access This article is licensed under a Creative Commons Attribution 4.0 International License, which permits use, sharing, adaptation, distribution and reproduction in any medium or format, as long as you give appropriate credit to the original author(s) and the source, provide a link to the Creative Commons licence, and indicate if changes were made. The images or other third party material in this article are included in the article's Creative Commons licence, unless indicated otherwise in a credit line to the material. If material is not included in the article's Creative Commons licence and your intended use is not permitted by statutory regulation or exceeds the permitted use, you will need to obtain permission directly from the copyright holder. To view a copy of this licence, visit http://creativecommons.org/licenses/by/4.0/. 
(Continued from previous page)

Conclusion: This study indicates that for ${ }^{177}$ Lu-DOTATATE PRRT, accurate estimates of absorbed dose for organs and tumors may be estimated from scans at $24 \mathrm{~h}, 72 \mathrm{~h}$, and 1 week post-treatment without an earlier scan. It may even be possible to cut out the 72 h scan, though the uncertainty increases. However, further work on more patients is required to validate this.

Keywords: Method dependence, Dosimetry, ${ }^{177}$ Lu-DOTATATE, Neuroendocrine tumors

\section{Introduction}

In recent years, peptide receptor radiotherapy (PRRT) using ${ }^{177}$ Lu-DOTATATE has been demonstrated to be effective for treatment of patients with metastatic neuroendocrine tumors (NETs) [1-3]. ${ }^{177}$ Lu-DOTATATE binds to somatostatin receptors present in NETs. ${ }^{177} \mathrm{Lu}$ decays mainly in the form of $\beta$ radiation, with a small part of $\gamma$ radiation. The $\beta$ radiation delivers effective radiotherapy to the immediately surrounding tissue, with little collateral damage to other organs. The $\gamma$ radiation (main energy $208 \mathrm{keV}$ ) permits post-treatment imaging that can be used to confirm that the radiopharmaceutical has reached its intended targets (primary tumor and/or metastases) and also for personalized dosimetry.

Personalized dosimetry calculations based on post-therapy SPECT images yield estimates of absorbed dose to tumors and normal tissues [4]. These absorbed dose estimates contribute to the planning of ongoing serial treatments, to avoid exceeding the absorbed dose threshold for vulnerable normal organs and to ensure sufficient absorbed dose to tumor, in the light of evidence of an absorbed dose-response curve, at least for pancreatic neuroendocrine tumors [5].

Absorbed dose to solid organs is essentially self-dose due to quite high activity within the organ, with a negligible contribution from cross-dose due to activity in other organs [6]. Total absorbed dose due to time-integrated activity concentration is given by the integral under the TACC multiplied with the appropriate factor (ACDF). While it is not practical to acquire images at more than 3-4 time points, it is also not possible to calculate an exact integral based on so few time points, and approximations must be made.

Others have addressed issues of reducing the number of post-treatment scans, even suggesting methods based on a single scan [7-11], but so far, the dependence on time points used for the suggested methods for dosimetry based on fewer scans has mostly been widely tested only for kidney dosimetry and not generally validated for normal organs and tumors. Furthermore, validation was not always performed with late time points (1 week) included in the reference method, as recommended by MIRD [12] to avoid substantial extrapolations in the calculation of absorbed dose. Our initial data demonstrated variability of the time course of ${ }^{177} \mathrm{Lu}$-DOTATATE in normal organs and to an even greater extent in tumors beyond that previously reported. This prompted us to investigate the time points as well as the numerical integration methods used to calculate absorbed dose and effective half-life, in the context of the observed diversity of TACCs including a late (1 week) time point.

This retrospective study examined TACCs for normal organs and tumors from patients receiving PRRT at our institution. To provide accurate estimates of absorbed dose to normal organs and tumors, while maintaining careful use of resources and not acquiring more images than necessary, we used the activity concentration data acquired 
in our current treatment protocol to investigate the following methodological questions: (i) impact of numerical integration method (single exponential fit, trapezoidal rule, or combination of these 2 methods) on estimates of absorbed dose and effective half-life; (ii) are 4 time points really required to obtain accurate dose estimates, or will 3 or even 2 points suffice, and if so which points can be omitted without compromising the results?

\section{Methods}

\section{Patients and treatment protocol}

Anonymized data from the first 30 patients who received ${ }^{177}$ Lu-DOTATATE PRRT and completed post-therapy imaging at our institution (Tel Aviv Sourasky Medical Center, Tel Aviv, Israel) were analyzed. Treatment protocols were as described in the literature $[2,13]$. In brief, patients received approximately $7.4 \mathrm{GBq}{ }^{177} \mathrm{Lu}$-DOTATATE in each treatment, diluted in saline and administered iv over a period of $30 \mathrm{~min}$ and also received an iv infusion of amino acids (L-lysine and L-arginine) as specified by the FDA [14], commencing $30 \mathrm{~min}$ before the start of the PRRT and continuing at least $3 \mathrm{~h}$ after, to reduce absorbed dose to kidneys.

Post-treatment imaging included SPECT/CT images approximately $4 \mathrm{~h}, 24 \mathrm{~h}, 4-5$ days, and 6-7 days after initiation of treatment. All images were acquired on a GE Optima NM/CT 640. SPECT images were acquired using a Medium Energy General Purpose (MEGP) collimator, acquiring 120 frames with a 30-s exposure time per frame (total acquisition time $30 \mathrm{~min})$. Coregistered CT was acquired on the four-slice CT $(140 \mathrm{kVp}, 3.0$ $\mathrm{mA}$ and half rotation) and used to generate an attenuation map. For SPECT reconstruction, the ordered subsets expectation maximisation (OSEM) algorithm included in the Xeleris 3.0 workstation (International General Electric, General Electric Medical Systems, Haifa, Israel) was used with default settings (iterative reconstruction with eight subsets and four iterations followed by a Hann filtering with a cut-off of 0.85). The images were attenuation corrected with the CT-created attenuation map.

IRB approval was given for this retrospective anonymized study.

\section{Image analysis}

Images were analyzed using the GE Dosimetry Toolkit to co-register serial SPECT/CT images, define VOIs for normal organs (left and right kidneys, spleen, liver) and for 34 tumor foci on all images, and calculate the corresponding TACCs. VOIs were defined on the SPECT images, with assistance of co-registered CT. VOIs were transferred automatically to all co-registered images in the series, with the option of manual user adjustment in case of need, as occurred particularly for tumor foci situated in soft tissue. In patients with tumor involvement in the liver, definition of VOIs including only normal liver was often challenging and limited to small sections of the liver.

\section{Dosimetry}

To permit conversion of data in counts to activity concentration the SPECT system was calibrated using phantom acquisitions including a known amount of activity in a known volume $[4,13]$ chosen to correspond to volumes typically used for VOIs defined for organs and tumors in the dosimetry. 
Absorbed dose for an organ or tumor is calculated by multiplication of timeintegrated activity concentration with the appropriate $\operatorname{ACDF}[4,15,16]$.

To calculate the time-integrated activity concentration, the TACC is typically described as mono- or bi-exponential washout of the injected activity from organs, and the integral is calculated directly from the parameters of the exponential(s). Biexponential washout includes a brief rapidly descending first phase, followed by a slower washout from there on. Frequently, the fast component of the washout is so brief that the data will not support a bi-exponential fit, and fitting the TACC to a single exponential yields a good approximation, and is therefore widely used for dosimetry, making it possible to base dose calculation on fewer time points. It was our original intention to use this option. However, with the first time point only $4 \mathrm{~h}$ post-injection, we often saw evidence of the initial fast decreasing exponential component, as reported by Delker et al. [17] for the case of kidney dosimetry, or, more surprisingly, an initial increase in activity apparently extending beyond $4 \mathrm{~h}$ post-injection before the curve started to decrease in mono-exponential washout.

As a result of this variability in initial behavior, inclusion of an early time point in a mono-exponential fit to estimate area under the curve may not always be accurate. Examples of this are shown in Fig. 1. To avoid rigid assumptions of the behavior of the curve during this initial time, we considered a combined method, estimating the integral under the curve as the sum of (i) trapezoidal integration from $t=0$ up to the second time point (approximately $24 \mathrm{~h}$ ) and (ii) the area under a mono-exponential fitted to time points 2,3 , and 4 (from $24 \mathrm{~h}$ on), extrapolated from injection out to infinity. We refer to this method as the "combined trapezoidal/mono-exponential" absorbed dose estimate. To decide which method to use as our "original method," we compared the combined method to the option simply to use all 4 time points for a monoexponential fit to all 4 time points. While mono-exponential fit of 4 standard time points also yielded a good fit for most normal organs and tumors, poor fit (as judged visually-for examples, see Fig. 1) as well as referring to the coefficient of determination $R^{2}$ (see the "Statistics" section below) was more frequent when all four time points were fitted to a mono-exponential than in the combined method where the early time point was not included in the mono-exponential fit. We therefore use the "combined trapezoidal/mono-exponential" method for estimating dose and half-life from 4 time points

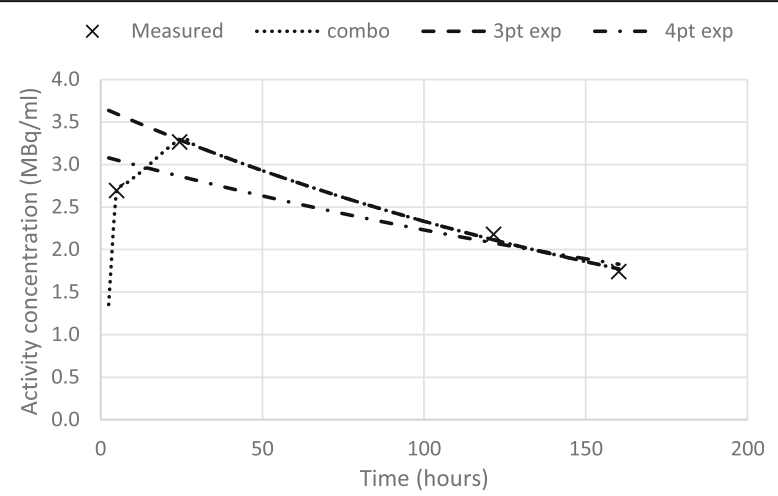

Fig. 1 Example illustrating the different extrapolations of the TACC used for calculation of absorbed dose and effective half-life. The trapezoidal rule from injection to $24 \mathrm{~h}$ is used in the "original" combined method, and curve fits to a 3- or 4-point mono-exponentials are shown 
as our reference method in this study and refer to it as our "original method." Note that in this "original method," points from $24 \mathrm{~h}$ on are fitted to a mono-exponential, and we considered the half-life based on this fit to characterize the long-term washout of ${ }^{177} \mathrm{Lu}$-DOTATATE. We compared this "original method" to the mono-exponential fit based on all 4 time points and to the alternative options of estimating dose using mono-exponential fit to 3 and 2 time point TACCs obtained by omitting time points.

\section{Statistics}

Time activity concentration curves (TACC's) of activity concentration, C, $(\mathrm{MBq} / \mathrm{ml})$ measured at 2,3, or 4 data points were fitted to a mono-exponential:

$$
C_{t}=C_{0} \times e^{-\ln (2) t / t_{e f f}}
$$

where $C_{\mathrm{t}}$ is the activity concentration at time $\mathrm{t}, C_{0}$ is the activity concentration at time zero obtained from the curve fit, and $t_{\text {eff }}$ is the effective half-life in therapy obtained from the curve fit.

Curve fitting was performed using the least squares method to obtain a fit to the activity concentrations, and the coefficient of determination $R^{2}$ was used as an indicator goodness of fit, with $R^{2}>0.95$ considered to be a good fit.

Note that both $C_{0}$ and $t_{\text {eff, }}$ representing initial uptake and half-life for washout, are crucial parameters in determining absorbed dose.

Bland-Altman plots, plotting percent differences in absorbed dose (or half-life) versus the mean values estimated using the original method and the method of choice, were used to compare the methods. To summarize the results of these comparisons, we also calculated the mean percent differences between each method and the "original method" and the 95\% confidence interval, indicating the uncertainty in these mean differences. To demonstrate the range of the differences between each method and the "original method" that may be expected over individual patients, we also calculated the 95\% percentile interval, taking into account both within and between patient variation for tumor foci and the kidneys and the proportion of tumors and organs with absolute percent difference $>10 \%$.

\section{Results}

Four SPECT/CT scans were acquired $5.0 \pm 0.7$ (mean \pm standard deviation), $23.4 \pm 1.0$, $112.9 \pm 13.0$, and $165.1 \pm 10.0 \mathrm{~h}$ post-treatment. VOIs were defined for normal organs and tumor foci for each patient, and time activity concentration curves (TACCs) were generated.

Visual inspection of the TACCs for normal organs and tumors confirmed the variability described in the "Methods" section. Specifically, while many TACCs followed a single exponential overall (Fig. 2a), in the initial 4-24 hours post treatment, a second more steeply decreasing exponential component (Fig. 2b) was evident in at least one TACC in 22 of 30 patients, or alternatively, an initial increase preceded the subsequent exponential decrease (Fig. 2c) in at least one TACC in 15 of 30 patients. TACCs obviously diverging from single exponential washout were, as suspected, seen most frequently in tumors but also in some normal organs. These visual observations together with the corresponding exponential fits with $R^{2}>0.95$ confirmed our decision to estimate absorbed dose using the combined trapezoidal/mono-exponential method described above (referred to here as the "original method"). 
a.

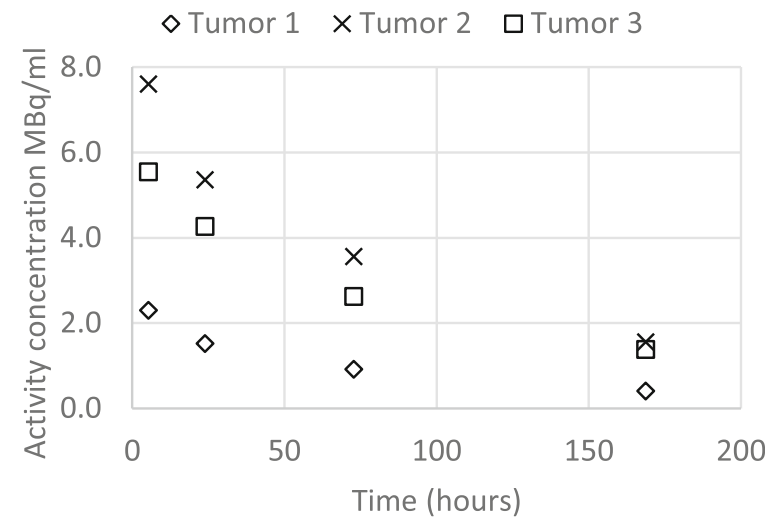

b.

$\times$ Tumor 1 aTumor $2 \diamond$ Tumor 3

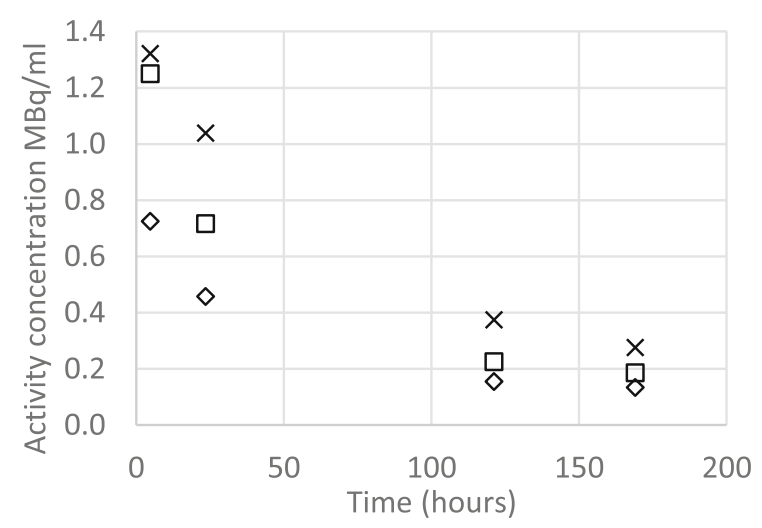

C.

$\times$ Tumor $1 \square$ Tumor $2 \diamond$ Tumor 3

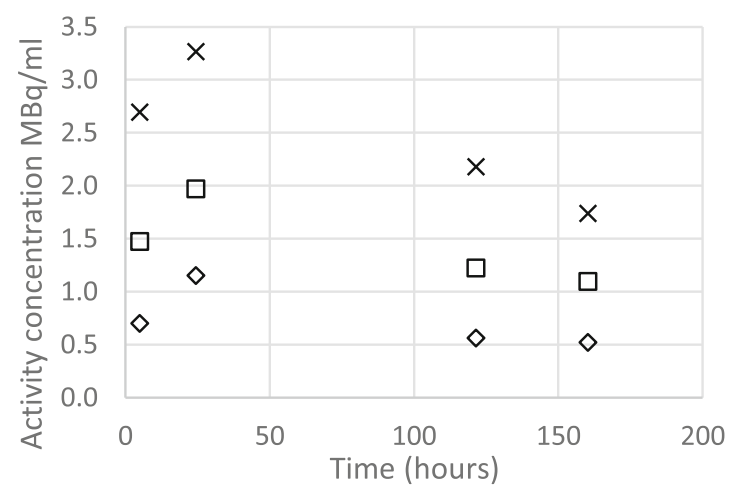

Fig. 2 Examples of time activity concentration curves for tumors, showing a mono-exponential decrease; $\mathbf{b}$ bi-exponential, with initial steeper decrease over first $24 \mathrm{~h}$ approximately, followed by mono-exponential decrease; c initial increase over first $24 \mathrm{~h}$ approximately, followed by mono-exponential decrease. Note that in all these graphs, tumors are labelled 1, 2, and 3 only to distinguish the curves from each other, and there is no significance to the numbering

\section{Absorbed dose and effective half-life for normal organs and tumors}

Absorbed dose and effective half-life estimates are shown in box-whisker plots in Fig. $3 a$ and $b$, respectively. These data indicate both higher absorbed dose and longer effective half-life for tumors as compared to normal organs, as expected. The mono- 

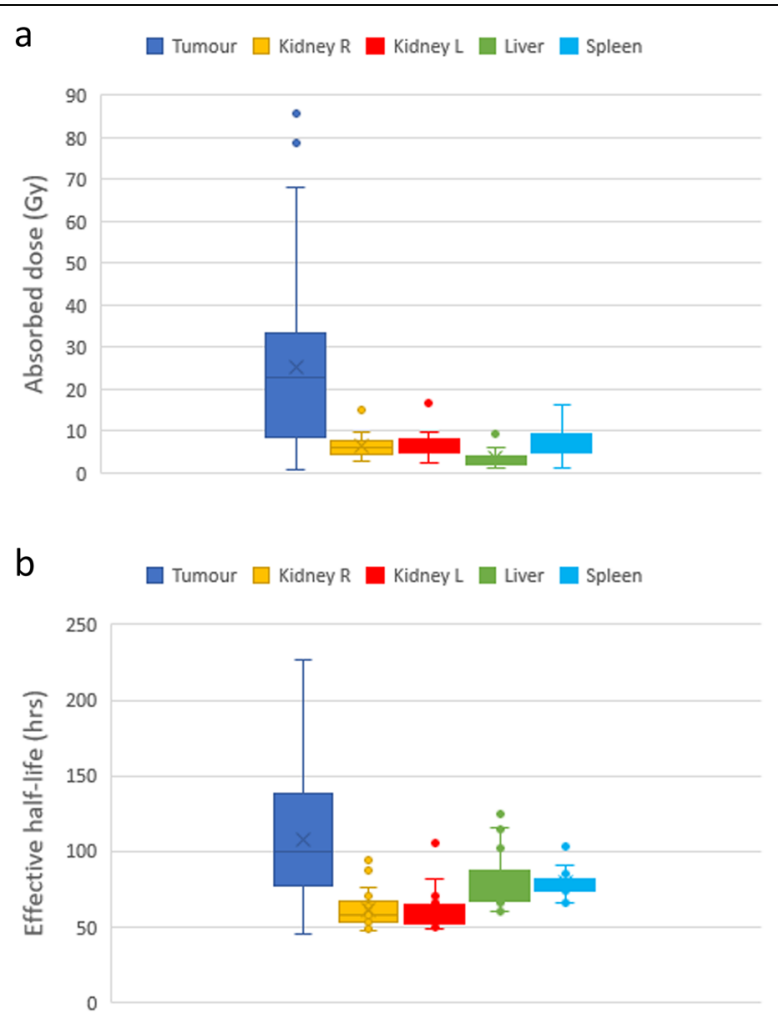

Fig. 3 Box-whisker plots for absorbed dose (a) and effective half-life (b) for the tumors, kidneys, liver, and spleen.

exponential fit of 3 standard time points ( $24 \mathrm{hr}, 4-5$ days, $6-7$ days) yielded a good fit $\left(R^{2}>\right.$ 0.95 ) for all normal organs ( $R$ and $L$ kidneys, liver, spleen) in $25 / 30$ patients and for all tumors in 21 patients; mono-exponential fit was somewhat less good $\left(0.90>R^{2}>0.95\right)$ for the remaining organs and tumors and failed for all three tumors in one patient. Data for these 3 tumors were not included in the comparisons between methods reported below.

Comparison of 4-time point mono-exponential fit from 0 to infinity vs "original method" The percentage differences in absorbed dose and effective half-life between the 4-point mono-exponential fits and the "original method" are shown in Bland-Altman plots (Fig. 4). The mean percent differences in estimated absorbed dose (Table 1) indicated that absorbed dose is on average slightly overestimated when using the 4 point exponential fit. However, mean differences were low for normal organs and only slightly higher (4.6\%) for tumors. Mean differences in effective half-life were also low ( $<3 \%)$ (Table 2). However, both over- and under-estimates of absorbed dose and effective half-life occurred, as reflected in the 95\% intervals; individual differences in absorbed dose were greater than $10 \%$ for 8 tumors, ranging up to $57 \%$, and differences in effective half-life were greater than $10 \%$ for at least one tumor or normal organ in 23 of the 30 patients, ranging up to $94 \%$, and exceeding $20 \%$ for at least one tumor or organ in 9 patients.

Visual examination of the observed data points together with the corresponding fitted curves, together with the values of the corresponding $R^{2}$, made it clear that the "original method" was a better match to the observed data than 4-time point mono-exponential 


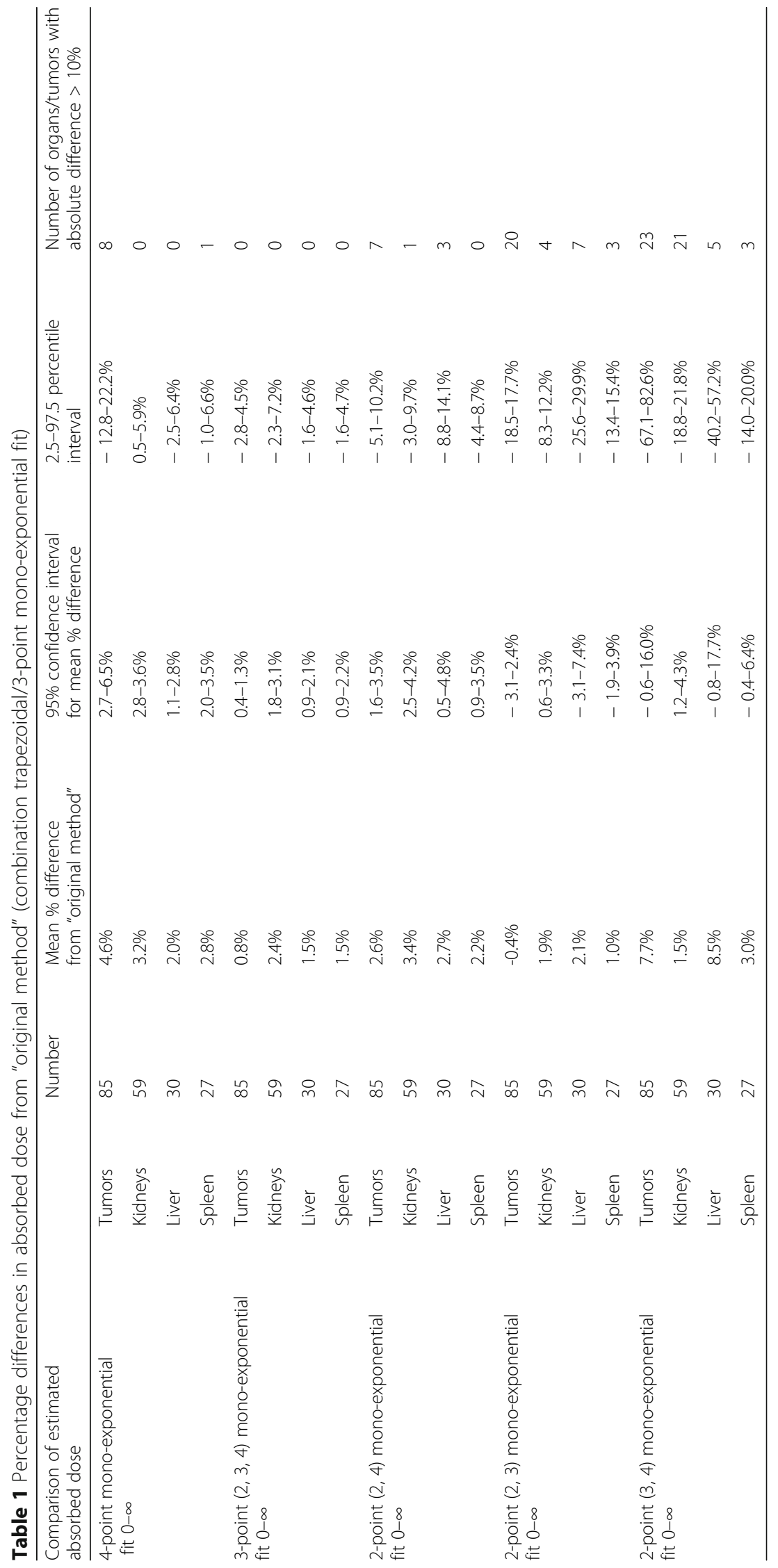




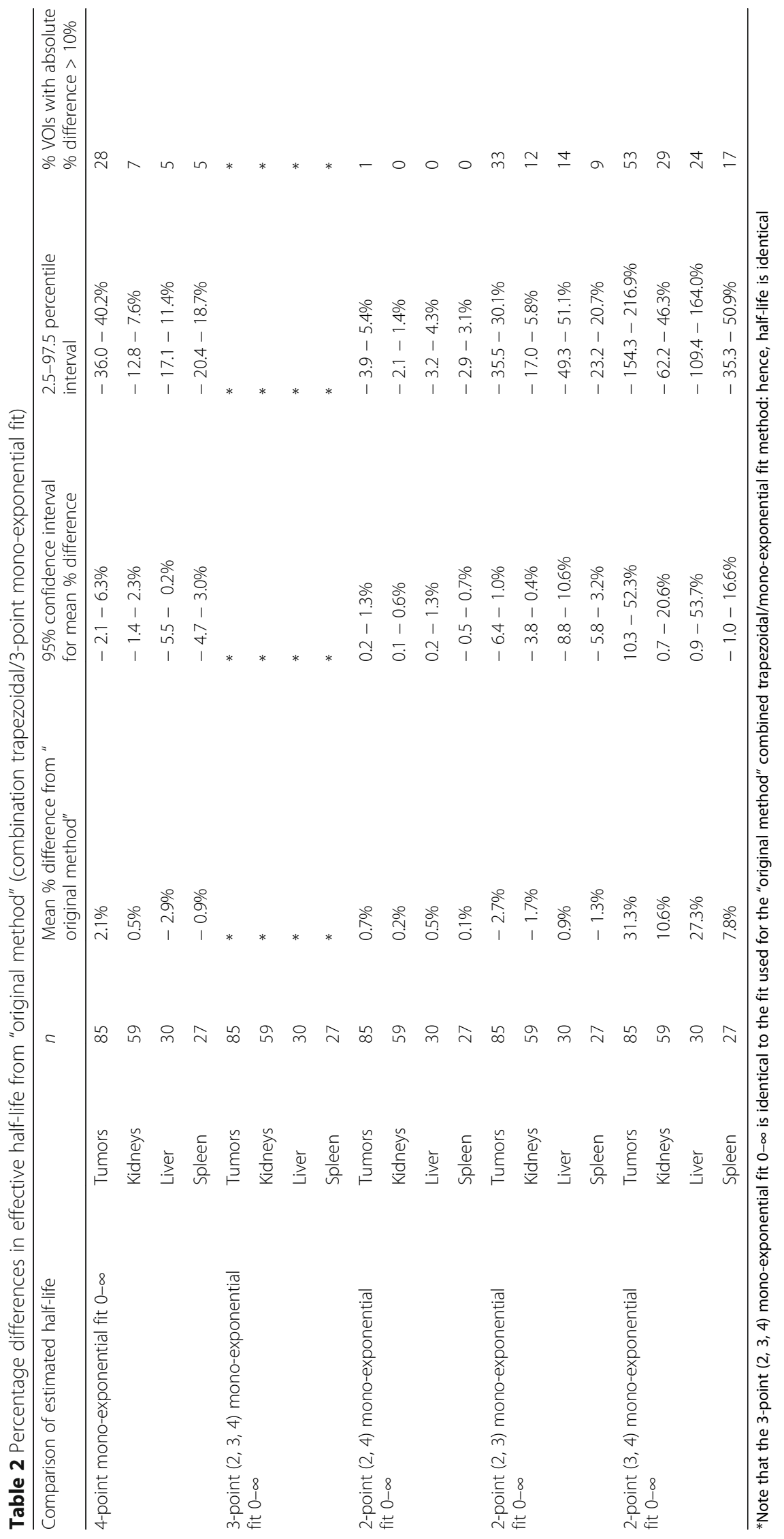




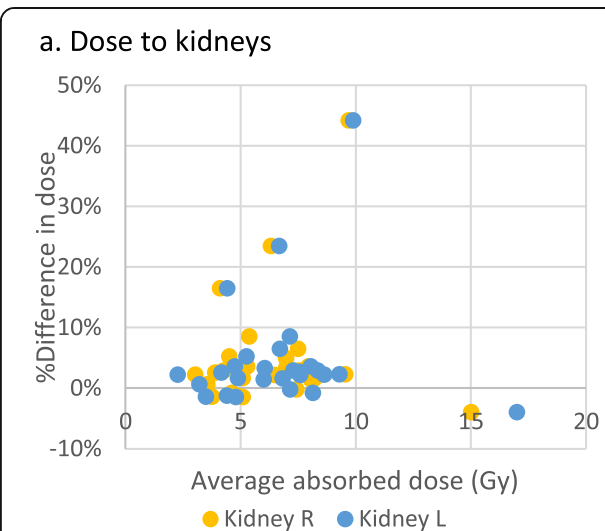

c. Dose to liver and spleen

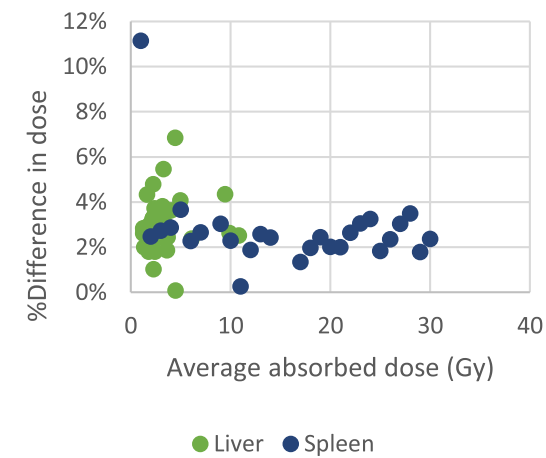

e. Dose to tumours

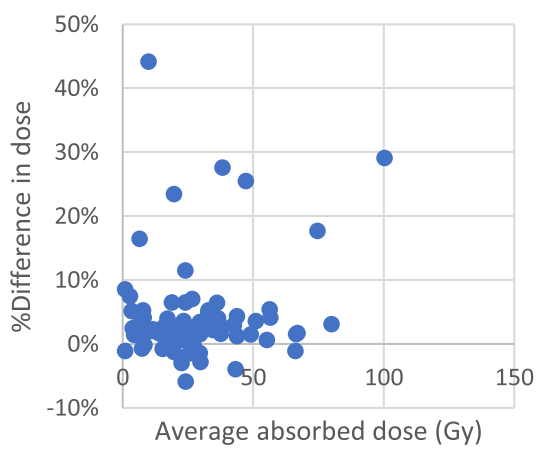

b. Half-life for kidneys

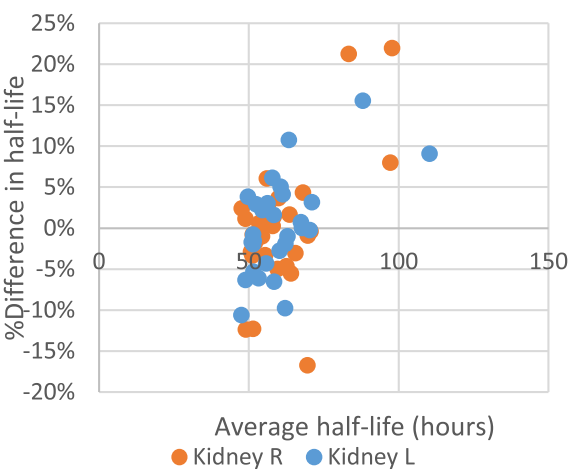

d. Half-life for liver and spleen

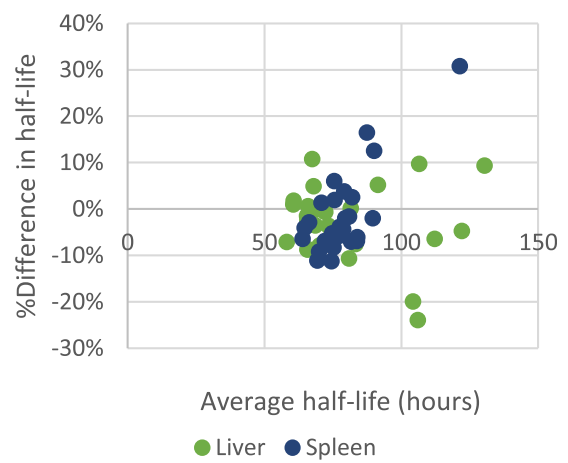

f. Half-life for tumours

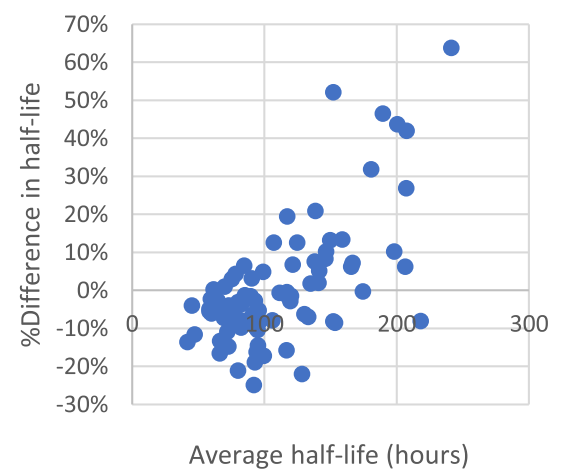

Fig. 4 Bland-Altman plots showing \% difference between 4-point mono-exponential fit and "original method" (combined trapezoidal/3-point-mono-exponential fit) for absorbed dose (a) and effective half-life (b) in the kidneys, liver and spleen (c and $\mathbf{d}$ ), and tumors (e and $\mathbf{f}$ ), plotted against average absorbed dose (Gy) and effective half-life (hours) for the two methods

fit from 0 to infinity. Errors in estimates of absorbed dose and effective half-life in the 4-point mono-exponential fit occurred most notably where initial uptake continued to increase after the early time point. In these cases, the discrepancy in estimated dose was not due to overestimating the integral in the first $24 \mathrm{~h}$, but to the skewed fit resulting from inclusion of the early time point yielding a longer half-life so that the tail end of the fitted curve is higher than its true time course. This comparison demonstrates the risks of including an early time point in the mono-exponential fit. While use of a 4point single exponential including a 4-h time point would give a fairly accurate absorbed dose for the majority of patient organs, and most tumors too; our findings 
suggest that it would give rise to a serious error in the absorbed dose for a minority of curves. Moreover, while discrepancy in estimated absorbed dose was only small for most tumors and organs, discrepancies in effective half-life for washout were more frequent and were greater than $10 \%$ in at least one tumor or organ in as many as 23 of the 30 patients.

\section{Comparison of $\mathbf{3}$ point exponential fit integration from $\mathbf{0}$ to infinity vs "original method"}

Discrepancies between absorbed dose based on the 3-point (24 and $96 \mathrm{~h}$ and 1-week post-treatment) mono-exponential fit versus our "original method" were all less than $5 \%$ for both tumors and normal organs. The mono-exponential fit in this case is identical to the exponential section of the "original method," so estimates of effective half-life are unchanged. The small discrepancies in absorbed dose due to the assumption of a mono-exponential curve from injection time included overestimates of absorbed dose in tumors or organs where activity concentration was initially slow to peak, and underestimates in the presence of an initial fast decaying exponential. But these differences were all small (Table 1), indicating that while using trapezoidal integration over the first $24 \mathrm{~h}$ may follow the true shape of the curve more closely than the mono-exponential fit; the difference in the total integral is small when considering resulting dose estimates.

Comparison of 2 point exponential fit integration from 0 to infinity vs "original method" Calculating absorbed dose and effective half-life based on pairs of scans (scans 2 and 4, scans 2 and 3, scans 3 and 4) yielded results that were highly dependent on which scans were used. Differences from our "original method" in estimates of absorbed dose and effective half-life are summarized in Tables 1 and 2, respectively. In brief, while a 3point mono-exponential fit is generally influenced most strongly by the first and last points, with relatively little effect of the middle time point, we were still surprised to find the differences in both absorbed dose and effective half-life to be consistently small when comparing mono-exponential fit of scans 2,3 , and 4 with the fit using only scans 2 and 4. On the other hand, mono-exponential fit to pairs of scans 2 and 3 or 3 and 4 gave rise to numerous larger differences in both absorbed dose and effective half-life, as reflected in very large 95 percent intervals, particularly when only scans 3 and 4 were used. These comparisons lead us to believe that dosimetry based on only scans 2 and 4 (24 $\mathrm{h}$ and 1-week post-therapy) could give accurate estimates of absorbed dose and effective half-life, whereas dosimetry based on only 2 scans at other pairs of time points could not necessarily be relied on. The 4-h time point was not included in these comparisons, since we already observed (see above) that it was often not representative of the mono-exponential fit out to infinity.

\section{Discussion}

In this retrospective study, we utilized time activity concentration curves formed from 4 serial scans acquired for dosimetry purposes to investigate how best to obtain accurate absorbed dose estimates from these scans, or better still, from an even smaller number of optimally timed post treatment scans. Scan acquisition is demanding in terms of resources and requires patient cooperation. 
Estimates of absorbed dose and effective half-life reported in this study are comparable to those reported by others $[4,5,13,18,19]$. In all but one of the 30 patients included in this study, absorbed dose to tumor was much higher than absorbed dose to normal organs, confirming high absorbed dose to tumors with relative sparing of normal tissue, as intended. Our calculations showed that higher tumor absorbed dose is due both to higher initial activity concentration (apparent on post-injection images) and longer effective half-life for activity in the tumor versus normal organs.

PRRT with ${ }^{177} \mathrm{Lu}$-DOTATATE is generally repeated at intervals of 5-12 weeks. For personalized dosimetry, a set of 3-4 post-therapy images may be acquired following the first treatment. It may be assumed that absorbed dose for the first treatment is a close approximation for absorbed dose on subsequent treatments. Alternatively, the approximation of constant effective half-life $[11,20]$ may be used. This makes it possible to obtain more accurate absorbed dose estimates for repeat treatments using a single scan 24-h post-treatment together with effective half-life from first treatment. When using this assumption, inaccuracy in effective half-life has implications for dosimetry for future treatments.

TACCs followed mono-exponential washout overall, but in some cases, the TACCs showed faster washout in the first hours, as reported by others [17], and in other cases an initial rise in activity concentration before subsequent mono-exponential decreases. To the best of our knowledge, this phenomenon of ongoing initial uptake has not been reported elsewhere, and its significance is unknown. Our data did not include sufficient time points to provide exact estimates of timing of peak activity, but approximate interpolation indicated that the peak could occur at least 8-9 h post injection.

In view of the observed diversity, we used combined trapezoidal/mono-exponential fit to estimate the integral under the curve. We did not attempt to fit the model of biexponential decrease, because there were TACCs for which this model was clearly unsuitable as described above. In addition, we were concerned that 4 data points would not be sufficient to yield an accurate fit of the larger number of parameters in this model. Further concerns relating to accuracy of the fit are that the exponential fits used relied on linear fit of the logarithms of the TACCs. Future work will include weighted fits as well as estimates of uncertainty of the absorbed dose and half-life and their implications for optimizing absorbed dose calculations for PRRT.

In our method comparisons, we first compared the two methods utilizing all 4 time points, our "original method" and the 4-point mono-exponential fit. Since we found that inclusion of the early $(4 \mathrm{~h})$ time point could skew the exponential fit, in our attempts to estimate absorbed dose from only 3 scans, we omitted the $4 \mathrm{~h}$ scan, and based absorbed dose estimates on the integral of the resulting 3-point monoexponential fit extrapolated from injection time to infinity, ignoring divergence from a mono-exponential in the first $24 \mathrm{~h}$ for some organs and tumors. This resulted in minimal differences in absorbed dose and effective half-life. Since the mean percentage of the total integral due to the activity concentration in the first $24 \mathrm{~h}$ post-injection was relatively low (mean $<23 \%$ ), as found also by others [21], it is reasonable that even quite large differences in contribution to absorbed dose from activity in this time period would only lead to small differences in total absorbed dose.

Investigating the possibility of basing dosimetry calculations on scans at only two time points, omission of the third ( $4-5$ day) time point, while not as accurate as using 3 
time points, resulted in fairly small differences in absorbed dose estimates and effective half-lives. A mono-exponential based on only 2 time points is entirely defined by the 2 points, a simple calculation with no test of goodness of fit, so with no possibility of checking the parameters subsequently used to calculate absorbed dose (from the area under the curve) and effective half-life (from the exponent). The potential impact of inaccuracy of the parameters on absorbed dose and effective half-life remains to be studied. It must also be remembered that organ dosimetry is based on definition of organ and tumor VOIs. When using 3-4 time point curves, sometimes low $R^{2}$ alerted us to error in human or automated VOI definition. Without such an indicator, appropriate VOI definition is even more crucial.

Thus, the results of this study suggest that the difference of estimated absorbed dose would not be large by cutting back from 4 to 3 post-treatment scans, omitting the $4 \mathrm{~h}$ scan, and that it might even be possible to rely on only 2 scans, $24 \mathrm{~h}$ and 7 days after treatment. Further work is required to confirm these results on a larger patient sample.

Others have addressed the question of minimum number and optimal timing of posttreatment scans required for dosimetry. Delker et al. [17] recommended that for kidney dosimetry after ${ }^{177} \mathrm{Lu}$-DOTATATE PRRT, measurements to be included in a monoexponential fit should be acquired more than 3-5 $\mathrm{h}$ after injection. Our findings are in agreement on this point and extend to tumors and additional normal organs. In addition, our findings suggest that it may be advisable to wait longer than $3-5 \mathrm{~h}$. While the MIRD recommendations [14] include imaging at a later time point, methods have been suggested that avoid this. Hanscheid et al. [7] propose that due to the relatively restricted range of effective half-life encountered, accurate dosimetry may be based on only one post-treatment scan 4 days after treatment. Our finding of considerable interpatient diversity in effective half-life casts doubt on whether this assumption can be extended to all organs and tumors. Others [8-11] have also studied options of estimating absorbed dose from PRRT based on only one scan, generally based on the assumption that there is little deviation from a population average time activity curve. This appears in discordance with the diversity in both absorbed dose and effective half-life observed in the 30 subjects in this study. In future work, a comparison to the methods proposed by these authors could be interesting. Our observation of difference in organ absorbed dose and effective half-life when the 1 week post-treatment scan is omitted also appears to contradict the recommendation that it is sufficient to scan only up to 3 days after PRRT $[4,11]$ for accurate dosimetry.

Future work is planned, before dispensing with the first and third post-treatment scans, to investigate other information in the 4-point TACCs, including whether the shape of the TACC in the first $24 \mathrm{~h}$ might be associated with specific tumor or disease characteristics, and/or implications for tumor response or toxicity in normal organs.

\section{Conclusion}

For personalized dosimetry in radionuclide therapy, dosimetry must be both accurate and accessible [22]. This study indicates that for ${ }^{177} \mathrm{Lu}$-DOTATATE PRRT, good estimates of absorbed dose and effective half-life for washout for organs and tumors may be estimated from scans at 24-h, 72-h and 1-week post-treatment without an earlier scan. It may even be possible to cut back to 2 post-treatment scans, at $24 \mathrm{~h}$ and 1 week. However, methods based on only 2 post-treatment scans would be even more user- 
dependent and require careful scrutiny of the VOIs. Further work on a larger number of patients is required to confirm these conclusions, and to check that other information would not be lost in reducing number of scans.

\section{Acknowledgements}

The authors wish to thank Professor Laurence S Freedman of Gertner Institute for Epidemiology and Health Policy Research, Sheba Medical Centre, Tel Hashomer, Israel for his statistical advice.

\section{Authors' contributions}

NF made absorbed dose calculations, drafted the manuscript, performed the statistical analysis, and conceived the study design. NS and $1 \mathrm{O}$ assisted with study design and execution. AS assisted with data collection and organization. JK and EE-S performed image interpretation for organ and tumor definition and helped with the draft of the manuscript. MS participated in the study design and helped to draft the manuscript. All authors read and approved the final manuscript.

\section{Funding}

No external funding was received for this work.

\section{Availability of data and materials}

The datasets used and/or analyzed during the current study are available from the corresponding author on reasonable request.

\section{Ethics approval and consent to participate}

IRB approval was given. The requirement of informed consent was waived for this retrospective anonymized study.

\section{Consent for publication}

We agree.

\section{Competing interests}

The authors declare that they have no competing interests.

\section{Author details}

${ }^{1}$ Institute of Nuclear Medicine, Tel Aviv Sourasky Medical Center, 6 Weizman Street, 64239 Tel Aviv, Israel. ${ }^{2}$ Section of Nuclear Medicine and PET, Department of Surgical Sciences, Uppsala University, Uppsala, Sweden. ${ }^{3}$ Institute of Radiotherapy, Tel Aviv Sourasky Medical Center, Tel Aviv, Israel. ${ }^{4}$ Sackler School of Medicine, Tel Aviv University, Tel Aviv, Israel.

Received: 13 December 2019 Accepted: 27 March 2020

Published online: 11 May 2020

\section{References}

1. Kwekkeboom DJ, de Herder WW, Kam BL, van Eijck CH, van Essen M, Kooij PP, Feelders RA, van Aken MO, Krenning EP. Treatment with the radiolabeled somatostatin analog [177 Lu-DOTA 0,Tyr3]octreotate: toxicity, efficacy, and survival. J Clin Oncol. 2008;26(13):2124-30.

2. Bodei L, Cremonesi M, Grana CM, et al. Peptide receptor radionuclide therapy with 177Lu-DOTATATE: the IEO phase I-II study. Eur J Nucl Med Mol Imaging. 2011;38:2125-35.

3. Strosberg J, El-Haddad G, Wolin E, Hendifar A, Yao J, Chasen B, Mittra E, et al. for the NETTER-1 Trial Investigators. Phase 3 trial of 177Lu-Dotatate for midgut neuroendocrine tumors. N Engl J Med. 2017;376:125-35.

4. Sandström M, Garske U, Granberg D, Sundin A, Lundqvist H. Individualized dosimetry in patients undergoing therapy with 177Lu-DOTA-D-Phe1-Tyr3-octreotate. Eur J Nucl Med Mol Imaging. 2010;37:212-25.

5. Ilan E, Sandström M, Wassberg C, Sundin A, Garske-Román U, Eriksson B, Granberg D, Lubberink M. Dose response of pancreatic neuroendocrine tumors treated with peptide receptor radionuclide therapy using 177Lu-DOTATATE. J NuCl Med. 2015:56:177-82.

6. Sandström M, Garske-Román U, Johansson S, Granberg D, Sundin A, Freedman N. Kidney dosimetry during 177LuDOTATATE therapy in patients with neuroendocrine tumors: aspects on calculation and tolerance. Acta Oncol. 2018 Apr; 57(4):516-21.

7. Hänscheid H, Lapa C, Buck AK, Lassmann M, Werner RA. Dose mapping after endoradiotherapy with 177Lu-DOTATATE/ DOTATOC by a single measurement after 4 days. J Nucl Med. 2018;59(1):75-81.

8. Willowson K, Eslick E, Ryu H, Poon A, Bernard EJ, Bailey DL. Feasibility and accuracy of single time point imaging for renal dosimetry following 177Lu-DOTATATE ('Lutate') therapy. EJNMMI Physics. 2018:5:33.

9. Madsen M, Menda Y, O'Dorisio TM, O'Dorisio MS. Single time point dose estimate for exponential clearance. Med. Phys. 2018:45:2318-24.

10. Zhao W, et al. Accuracy of kidney dosimetry performed using simplified time activity curve modelling methods: a 177Lu-DOTATATE patient study. Phys. Med. Biol. 2019;64:175006.

11. Sundlöv A, Gustafsson J, Brolin G, Mortensen N, Hermann R, Bernhardt P, Svensson J, Ljungberg M, Tennvall J, Sjögreen GK. Feasibility of simplifying renal dosimetry in 177Lu peptide receptor radionuclide therapy. EJNMMI Phys. 2018;5(1):12.

12. Siegel JA, Thomas SR, Stubbs JB, Stabin MG, Hays MT, Koral KF, Robertson JS, Howell RW, Wessels BW, Fisher DR, Weber DA, Brill AB. MIRD pamphlet no. 16: Techniques for quantitative radiopharmaceutical biodistribution data acquisition and analysis for use in human radiation dose estimates. J Nucl Med. 1999;40(2):37S-61S. 
13. Garske-Román U, Sandström M, Fröss Baron K, Lundin L, Hellman P, Welin S, Johansson S, Khan T, Lundqvist H, Eriksson B, Sundin A, Granber D. Prospective observational study of 177Lu-DOTA-octreotate therapy in 200 patients with advanced metastasized neuroendocrine tumors (NETs): feasibility and impact of a dosimetry-guided study protocol on outcome and toxicity. EJNMMI. 2018;45:970-88.

14. https://www.drugs.com/mtm/lutathera.html I. Accessed 18 March 2020

15. Sandström M, llan E, Karlberg A, Johansson S, Freedman N, Garske-Román U. Method dependence, observer variability and kidney volumes in radiation dosimetry of 177Lu-DOTATATE therapy in patients with neuroendocrine tumors. EJNMMI Physics. 2015;2:24.

16. Zaknun JJ, Bodei L, Mueller-Brand J, Pavel ME, Baum RP, Hörsch D, O'Dorisio MS, O'Dorisiol TM, Howe JR, Cremonesi M, Kwekkeboom GJ. The joint IAEA, EANM, and SNMMI practical guidance on peptide receptor radionuclide therapy (PRRNT) in neuroendocrine tumors. Eur I Nucl Med Mol Imaging. 2013;40:800-16.

17. Delker A, Ilhan H, Zach C, Brosch J, Gildehaus FJ, Lehner S, Bartenstein P, Böning G. The Influence of early measurements onto the estimated kidney dose in [177Lu][DOTA0,Tyr3] octreotate peptide receptor radiotherapy of neuroendocrine tumors. Mol Imaging Biol. 2015;17:726-34

18. Sandström M, Garske-Román U, Granberg D, Johansson S, Widström C, Eriksson B, Sundin A, Lundqvist H, Lubberink M. Individualized dosimetry of kidney and bone marrow in patients undergoing 177Lu-DOTA-Octreotate treatment. J NuCl Med. 2013:54:1-9.

19. Cremonesi M, Botta F, Di Dia A, Ferrari M, Bodei L, De Cicco C, Rossi A, Bartolomei M, Mei R, Severi S, Salvatori M, Pedroli G, Paganelli G. Dosimetry for treatment with radiolabelled somatostatin analogues. A review. Q J Nucl Med Mol Imaging. 2010 Feb;54(1):37-51.

20. Garske U, Sandström M, Johansson S, Sundin A, Granberg D, Eriksson B, Lundqvist H. Minor changes in effective half-life during fractionated 177 Lu-Octreotate therapy. Acta Oncologica. 2012;51:86-96.

21. Sandstrom M, Garske-Roman U, Granberg D, Sundin A, Lubberink M. Fractional contribution of extrapolations in absorbed dose calculation to kidneys in 400 patients with neuroendocrine tumors receiving 177Lu-DOTATATE therapy. EJNMMI. 2014;41(Suppl 2):S151-705 OP294

22. Giammarile F, Muylle K, Delgado Bolton R, Kunikowska J, Haberkorn U, Oyen W. Dosimetry in clinical radionuclide therapy: the devil is in the detail. Eur J Nucl Med Mol Imaging. 2017 Nov;44(12):2137-9.

\section{Publisher's Note}

Springer Nature remains neutral with regard to jurisdictional claims in published maps and institutional affiliations.

\section{Submit your manuscript to a SpringerOpen ${ }^{\circ}$ journal and benefit from:}

- Convenient online submission

- Rigorous peer review

- Open access: articles freely available online

High visibility within the field

- Retaining the copyright to your article

Submit your next manuscript at $\boldsymbol{\nabla}$ springeropen.com 